\title{
Epidemiology and health services research in 1996
}

Perhaps the most notable change in the papers submitted to this journal over the first few years has been the increase in what can be generally described as health services research. About six papers in this category will appear in this issue, the first being part of the Cochrane Collaboration looking at the contents of this Journal itself over the years since it began. A crude average of two randomised controlled trials (RCTs) published per year since we began gives no indication of the rapid upward trend, to five per year in the last five year period studied. One RCT is published in the current number, in the field of health promotion which surely needs more evaluation to enable resources to be better focused.

Two other papers describe cost effectiveness studies, one in prevention and one in orthopaedic surgery.

Analytical epidemiology continues to hold an important place in the journal, with a wide variety of topics and some papers on methods. However, a large number of descriptive epidemiology papers are received of which only a modest proportion are accepted. It is true that many topics of importance to the public health have been so little researched that descriptive studies are needed. But a journal such as this will always incline (readers and would-be authors will understand) towards papers which test hypotheses.

One powerful approach for use in both analytical and descriptive epidemiology is the person-years approach using what Case called the quinary-quinquennial cohort group. All modern epidemiologists will have used this powerful approximation - approximate essentially because information is not usually available by a single year of age and time period.

The late Martin Gardner from Southampton first pointed out that strictly the "cohort" names should span part of two years (that is, should be eg 1990-91 rather than 1990) and developed the concepts further (with more powerful computing tools) with Clive Osmond who has written a comment on the article by Case which we have reproduced in this number.

We look forward to continuing with the publication of applied epidemiology or, as a colleague on our Editorial Board once called it, "useful epidemiology". Health services research can progress only as fast as colleagues apply thoughtful and rigorous techniques - including but broader than classical epidemiology - to problems in our environment and our health services which affect the health of us all.

\section{Referees}

AUTHORS TO SUGGEST SUITABLE REFEREES

This journal uses a peer review system for appraising and improving submitted articles. Reviewers assess manuscripts by considering the importance of the research question, the study's scientific strength, the written presentation and the paper's appropriateness for this journal.

Many authors have taken up the recent suggestion that, when they submit papers for publication, they should give names and addresses of four experts whom they consider competent and suitable to review their work. The Editor makes no commitment to use these nominated referees, but will make an independent decision - some will be used to advise on the suitability of papers. I recommend that all authors should put forward up to four names of suitable reviewers.

STUART DONNAN Editor 